\title{
Entwicklung eines Generators zur Energieversorgung von Biosensoren
}

\author{
T. Reuter $^{1}$, M. Beck ${ }^{1}$, S. Liebold ${ }^{1}$, M. Hoffmann ${ }^{1}$, P. Hörschelmann ${ }^{2}$, E. Weisheit ${ }^{2}$, \\ S. Schmidt ${ }^{3}, T$. Bartsch ${ }^{3}$ \\ ${ }^{1}$ fzmb GmbH, Geranienweg 7, 99947 Bad Langensalza, Deutschland, \\ treuter@fzmb.de \\ ${ }^{2}$ Jub - Creative Product GmbH, Industriestraße 12, 99846 Seebach, Deutschland \\ ${ }^{3}$ BITSz engineering $\mathrm{GmbH}$, Newtonstraße 12, 08060 Zwickau, Deutschland
}

\begin{abstract}
Abstrakt:
Aktuelle Forschungsarbeiten am fzmb beschäftigen sich mit der Entwicklung energieautarker Sensorsysteme für die Anwendung an Tier und Mensch [1]. Dabei wurde ein Energiegenerator entwickelt, der in der Lage ist, aus der Bewegungsenergie von Tieren und Menschen Energie für Sensoren und Telemetriesysteme bereitzustellen. Die Funktion des Generators beruht auf der Verwendung eines Rotationspendels, dessen Energie in einem Drehfedermechanismus zwischengespeichert wird, welches wiederum über eine momentgesteuerte Kupplung die zwischengespeicherte Energie auf den Generator überträgt. Die Erprobung des Energiegenerators erfolgte im Pansen eines Rindes [2], fixiert am Bauch eines Menschen und Pferdes. Die erreichten Leistungsdaten liegen im $\mu \mathrm{W}$ - bis mW-Bereich und dienen als erste Abschätzung für die mögliche Unterstützung der Energieversorgung von Lowpower Anwendungen. Die hier angedachten Lowpower Anwendungen sind Sensoren für physiologische Parameter und Kurzstreckentelemetrie.
\end{abstract}

Key words: Energy Harvesting, Energiegenerator, Bewegungsenergie, autarke Energieversorgung

\section{Einleitung}

Im Bereich der industriellen Landwirtschaft werden seit Jahren verstärkt Systeme nachgefragt, die objektive Daten über Gesundheits-, Fruchtbarkeits- und Verhaltenszustände von Nutztieren liefern. Die Akzeptanz solcher Systeme ist grundsätzlich von deren praktischer Anwendbarkeit abhängig. In diesem Zusammenhang ist die Energieversorgung ein außerordentlich kritischer Punkt. Daher beschäftigen sich aktuelle Forschungsarbeiten am fzmb mit der Entwicklung energieautarker Sensorsysteme für die Anwendung an Tier und Mensch. Dabei wurde ein Energiegenerator entwickelt, der in der Lage ist, aus der Bewegungsenergie von Tieren und Menschen Energie für Sensoren und Telemetriesysteme bereitzustellen. Die Erprobung des Energiegenerators wurde im Pansen eines Rindes durchgeführt. Des Weiteren wurde der Energiegenerator am Menschen und am Pferd jeweils am Applikationsort Bauch auf einem Laufband mit einer Geschwindigkeit von $1,5 \mathrm{~m} / \mathrm{s}$ getestet. Die erreichten Leistungsdaten dienen als erste Abschätzung für die Unterstützung der Energieversorgung von Lowpower Anwendungen. Die hier angedachten Lowpower Anwendungen beziehen sich auf die
Sensoren für die Messungen physiologische Parameter (Pansenbewegungen) und deren Übermittlung an einem externen Empfänger mittels Kurzstreckentelemetrie.

\section{Energy Harvesting}

Die Gewinnung kleiner Mengen von elektrischer Energie aus Quellen wie Umgebungstemperatur, Vibrationen oder Luftströmungen für mobile Geräte mit geringer Leistung bezeichnet man als Energy Harvesting [3]. Je nach Energieform stehen für das Energy Harvesting verschiedene physikalische Effekte zur Verfügung. Die Tabelle 1 gibt einen Überblick über die wesentlichen Energieformen und die möglichen physikalischen Effekte.

Tab. 1: Übersicht über verschiedene Energieformen und physikalische Effekte [3, 4].

\begin{tabular}{|l|l}
\hline Energieform & Physikalischer Effekt \\
\hline Kinetisch & $\begin{array}{l}\text { Faradaysches Induktionsprinzip, } \\
\text { piezoelektrischer und pyroelektrischer Effekt, } \\
\text { Coulomb'sches Gesetz }\end{array}$ \\
\hline Thermisch & Seebeck und Peltier Effekt \\
\hline Licht & Photoeffekt \\
\hline Chemisch & Elektrochemische katalytische Oxidation \\
\hline
\end{tabular}

Die Grundlage des hier zu entwickelnden energieautarken Sensorsystems ist die Umwandlung von mechanischer in elektrische Energie 
mittels eines Generators, der auf dem Prinzip der faradayschen Induktion beruht. Wesentlich für die Generierung von Leistungen sind die im Pansen oder an Probanden (Mensch oder Tier) auftretenden Beschleunigungen. Weiterhin entscheidend ist der jeweilige Applikationsort des Energiegenerators am Probanden.

\section{Physiologie der Tier- und Pansenbewegung}

Aufgrund der sich für landwirtschaftliche Nutztiere stetig in Verbesserung befindlichen Haltungsbedingungen ist es immer mehr Rindern möglich sich in Ställen ohne Anbindehaltung oder auf der Weide frei zu bewegen. Die anatomische Beschaffenheit des Gastointestinaltraktes (GIT) der heutigen Wiederkäuer repräsentiert das Ergebnis eines langen Adaptationsprozesses an cellulosereiches Futter. Wiederkäuer entwickelten im Laufe der Evolution ein Vormagensystem bestehend aus Fermentationskammern (siehe Abb.1). Funktionell bilden Haube und Pansen eine Einheit und werden deshalb auch als Reticulorumen bezeichnet. Durch die Hauben-Pansen-Motorik wird das grob gekaute Futter nach dem Abschlucken nach dorsal geschoben und befindet sich dann im dorsalen Pansensack. Das Wiederkauen führt zur Abnahme der Partikelgröße und Zunahme der Partikeldichte. Die Partikel gelangen durch die Kontraktion des ventralen Pansensackes in den Pansenvorhof und anschließend in die Haube. Die Vormagenmotorik ermöglicht eine intensivere Durchmischung der Ingesta, die Abgabe der Pansengase (Ruktus) und den geregelten Weitertransport von Ingesta aus dem Reticulorumen in den Psalter (Blättermagen). Beim gesunden Tier finden etwa drei Kontraktionen innerhalb von zwei Minuten statt. Während der Futteraufnahme ist die Frequenz nahezu doppelt so hoch $[5,6]$.

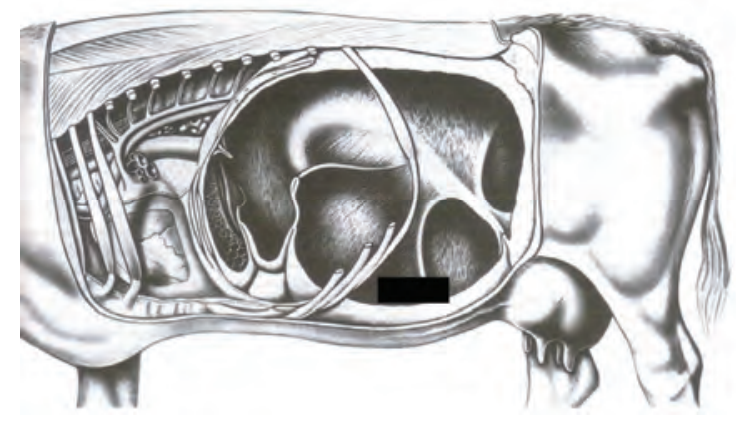

Abb. 1 Pansen [7] mit Lage der Pansensonde (schwarzer Balken).

Aufgrund der unterschiedlichen Gangphysiologie von Pferd und Mensch wurde die Möglichkeit des Energy Harvesting bei einer Laufbandgeschwindigkeit von $1,5 \mathrm{~m} / \mathrm{s}$ getestet, was beim Pferd einem leichten Trab und beim Menschen dem zügigen joggen entspricht.

\section{Funktion Energiegenerator}

Die Funktion des Generators beruht auf der Verwendung eines Rotationspendels, dessen Energie in einem Drehfedermechanismus zwischengespeichert wird, welches wiederum über eine momentgesteuerte Kupplung die zwischengespeicherte Energie auf den Generator überträgt (siehe Abb. 2).

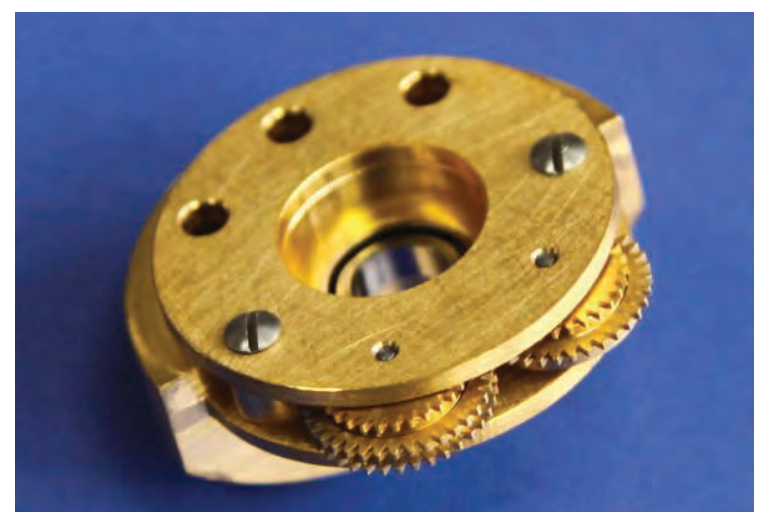

Abb. 2. Baueinheit: Platine, Kupplungen und Lagerung.

Da die von Tier oder Mensch erzeugte Bewegungsenergie bezüglich Betrag und Richtung zufällig verteilt ist, wurde ein Richtgetriebe entwickelt. Um einen möglichst großen Anteil der Bewegungsenergie verwenden zu können, nutzt dieses Richtgetriebe die Bewegungsimpulse beider Rotationsrichtungen zum Spannen der Schlingfeder. Es wurde ein Schlingfedermechanismus entwickelt, welcher den relativ schmalen Drehzahlbereich, in dem der Generator einen guten Wirkungsgrad hat, ausnutzt. Diese Anordnung erlaubt es, dass sich die gesamte aufgebaute Federspannung mit einem Mal überträgt und dabei in einem optimalen Drehzahlbereich bleibt (siehe Abb. 3).

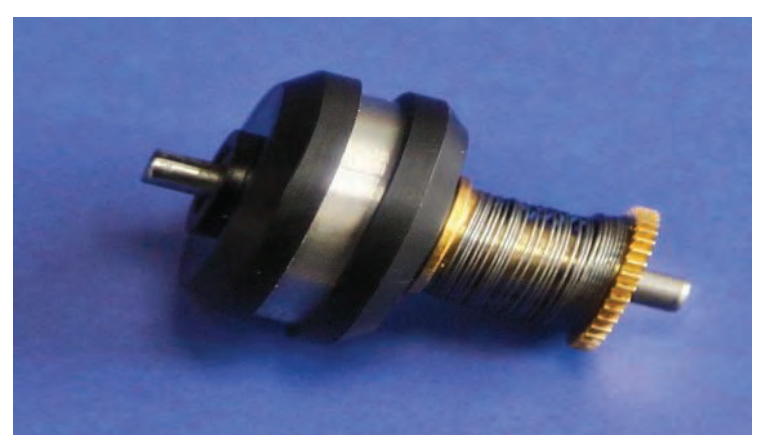

Abb. 3. Montierte Generator Spiralfederbaugruppe.

Als Generator wird eine permanentmagneterregte Synchronmaschine verwendet [8]. Aufgrund der unterschiedlich auftretenden Beschleunigungen für die Anwendung im Pansen (Studie A) und für die Anwendung an Mensch und Pferd (Studie B und C) wurden zwei Energiegeneratoren entwickelt. Der Energiegenerator der Studie A wurde in Form eines Pansen- 
bolus konstruiert (siehe Abb. 4) Die Abmaße des Energiegenerators sind $35 \mathrm{~mm}$ im Durchmesser und $40 \mathrm{~mm}$ in der Länge. Die Masse beträgt $140 \mathrm{~g}$.

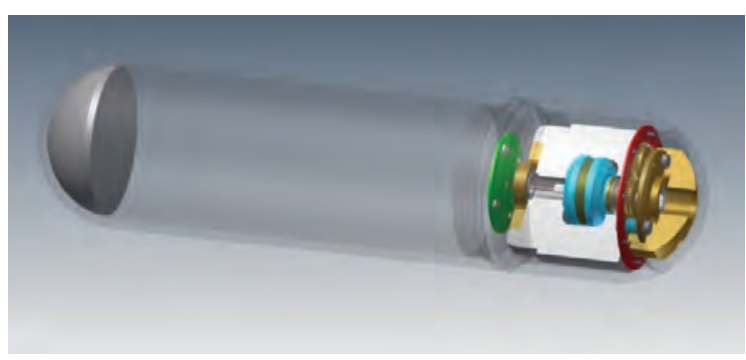

Abb. 4. Konstruktionsdarstellung des Energiegenerators in Form eines Pansenbolus (Studie A).

Für die Studie B und C wurde der Energiegenerator in Form eines zylindrischen Gehäuses mit den Abmaßen: $52 \mathrm{~mm}$ im Durchmesser und $31 \mathrm{~mm}$ in der Länge konstruiert (siehe Abb. 5). Die Masse beträgt $50 \mathrm{~g}$.

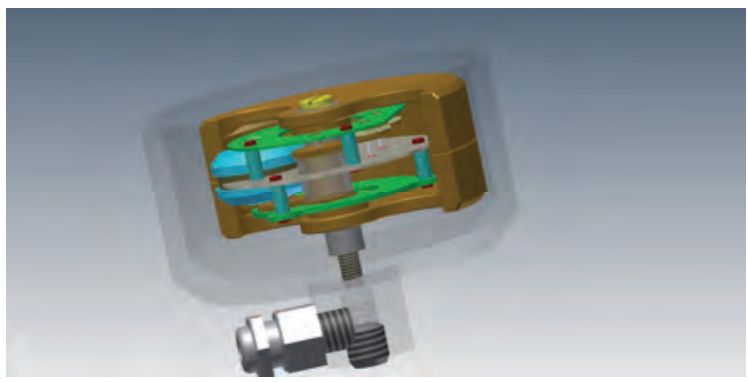

Abb. 5. Konstruktionsdarstellung des Energiegenerators in Form eines zylindrischen Gehäuses (Studie $B$ und $C$ ).

\section{Berechnung der generierten Leistung}

Die Berechnung der generierten Leistungen $\mathrm{P}$ in $\mathrm{mW}$ erfolgte nach Gleichung 1:

$$
P=W \cdot \frac{a}{t}
$$

Dabei entspricht W der Energiemenge (Arbeit) die der Generator bei einer Entladung abgibt. Die Energiemenge wurde mittels Voruntersuchungen bestimmt und beträgt pro Entladung $3.3 \mathrm{mWs}$ (Studie A) bzw. $0.65 \mathrm{mWs}$ (Studie B und $\mathrm{C}$ ). In Gleichung 1 gibt der Parameter a die Anzahl der Entladungen pro Versuchszeit und $t$ die Versuchsdauer an.

\section{Experimentelle Versuche}

Die Erprobung des Generators erfolgte im Pansen eines Rindes mit einer Versuchszeit von 25 h (Studie A). Dabei wurde der Generator in einem zylinderförmigen Gehäuse befestigt und über eine zuvor chirurgisch hergestellte Pansenfistel in den ventralen Pansensack eingegeben (siehe Abb. 6). Der Pansenbolus (zylinderförmiges Gehäuse) hatte eine Dichte von
2,3 $\mathrm{g} / \mathrm{cm}^{3}$. Damit wurde sichergestellt, dass der Bolus sich nur im ventralen Pansensack befindet und nicht von der Pansenmotorik in die anschließenden Vormägen transportiert wird (siehe Abb. 1). Des Weiteren wurde der Generator am Mensch (Studie B) und am Pferd (Studie C) getestet.

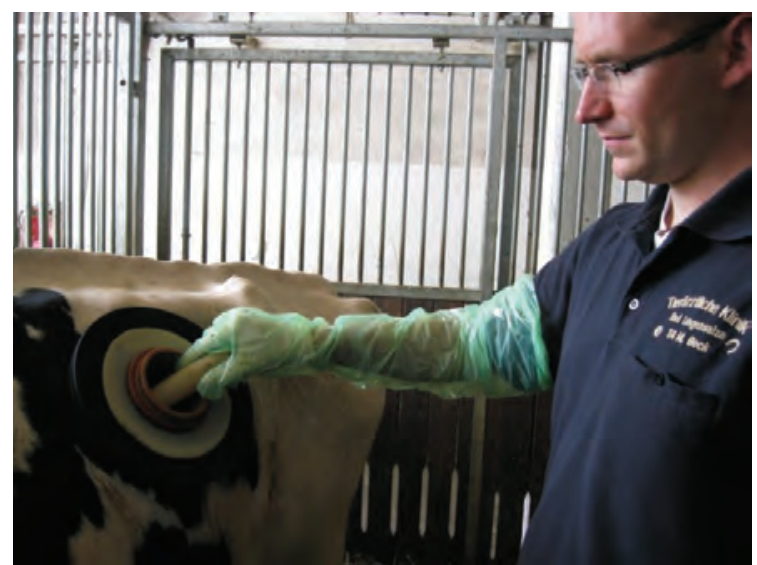

Abb. 6. Eingabe des Pansenbolus über eine Fistel.

Bei den Studien B und C wurde der Generator mittels Tragegurt am Bauch des jeweiligen Probanden befestigt (siehe Abb. 7). Dabei wurde die Anzahl der Entladungen bei einer konstanten Laufgeschwindigkeit von 1,5 m/s (Laufband) innerhalb einer Versuchszeit von $300 \mathrm{~s}$ registriert. Bei allen drei Studien wurde ebenfalls die auftretende Beschleunigung in $x-, y-$ und $z-$ Richtung am Applikationsort des Energiegenerators (im Pansen und am Bauch) registriert.

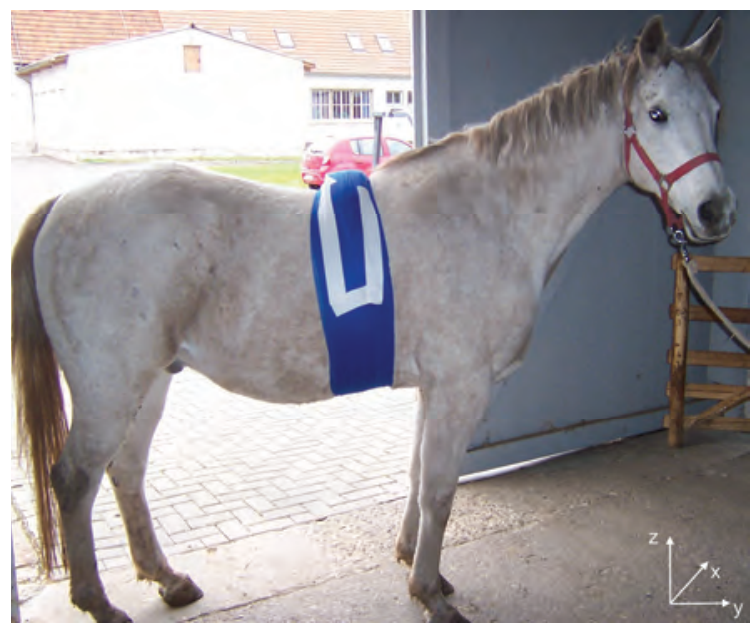

Abb. 7 Befestigung des Energiegenerators am Bauch (Tragegurt mit blauer Bandage fixiert) eines Pferdes.

\section{Resultate}

Die Ergebnisse sind in den Abbildungen 8 (Studie A), 9 (Studie B) und 10 (Studie C) zusammenfassend dargestellt. Die durchschnittlichen Beschleunigungen betrugen $9.03 \mathrm{~m} / \mathrm{s}^{2}$ (Studie A), $10,8 \mathrm{~m} / \mathrm{s}^{2}$ (Studie B) und $9,9 \mathrm{~m} / \mathrm{s}^{2}$ (Studie C). Dabei wurden 207 Entladungen (Studie A), 
50 Entladungen (Studie B) und 68 Entladungen (Studie C) vom Generator während der Versuchszeit registriert. Dies entsprach nach Glei- chung 1 einer Leistung von $8 \mu \mathrm{W}$ (Studie A), $0,11 \mathrm{~mW}$ (Studie B) und 0,15 $\mathrm{mW}$ (Studie C) bezogen auf die jeweilige Versuchszeit.
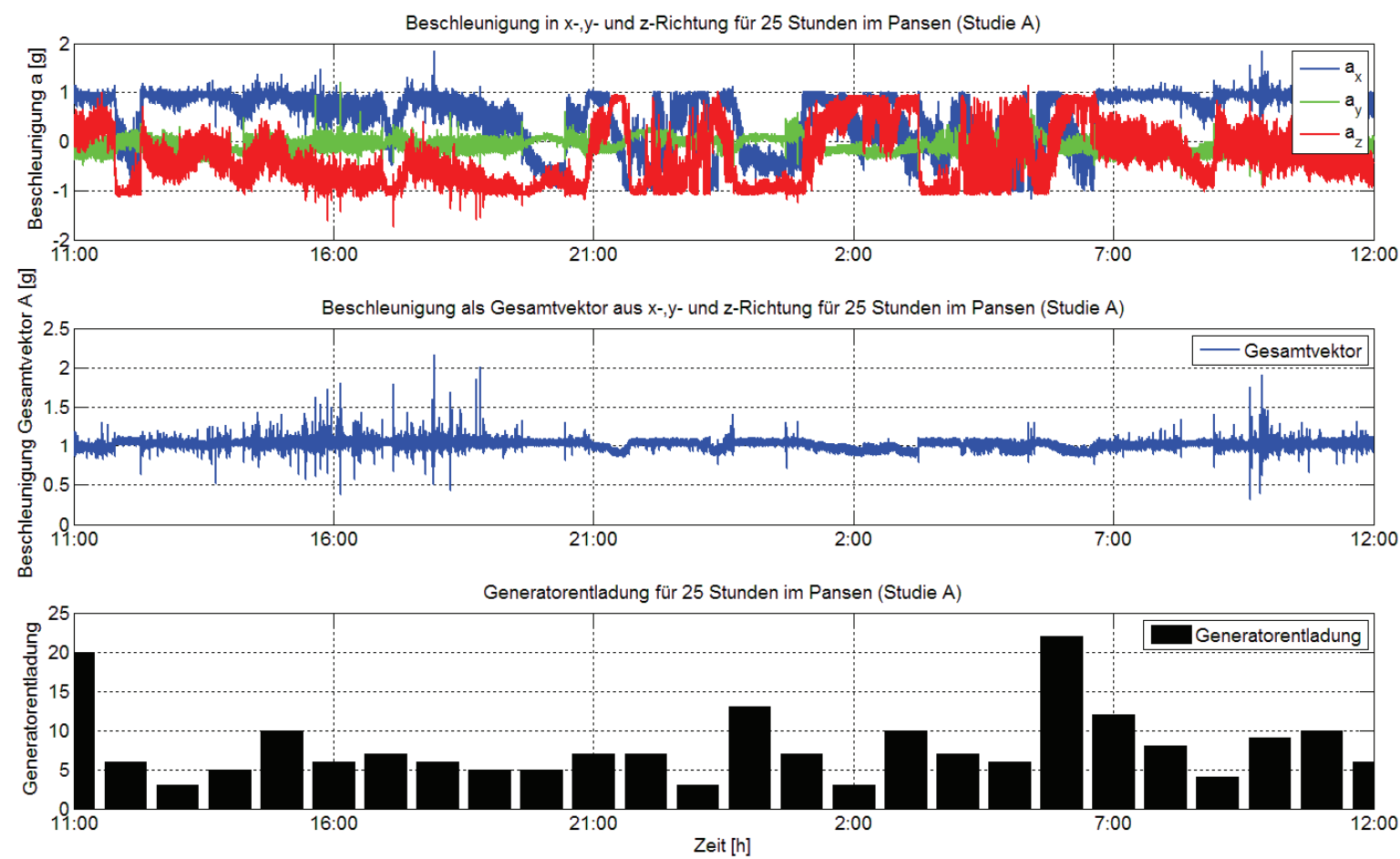

Abb. 8. Experimentelle Daten Studie A.
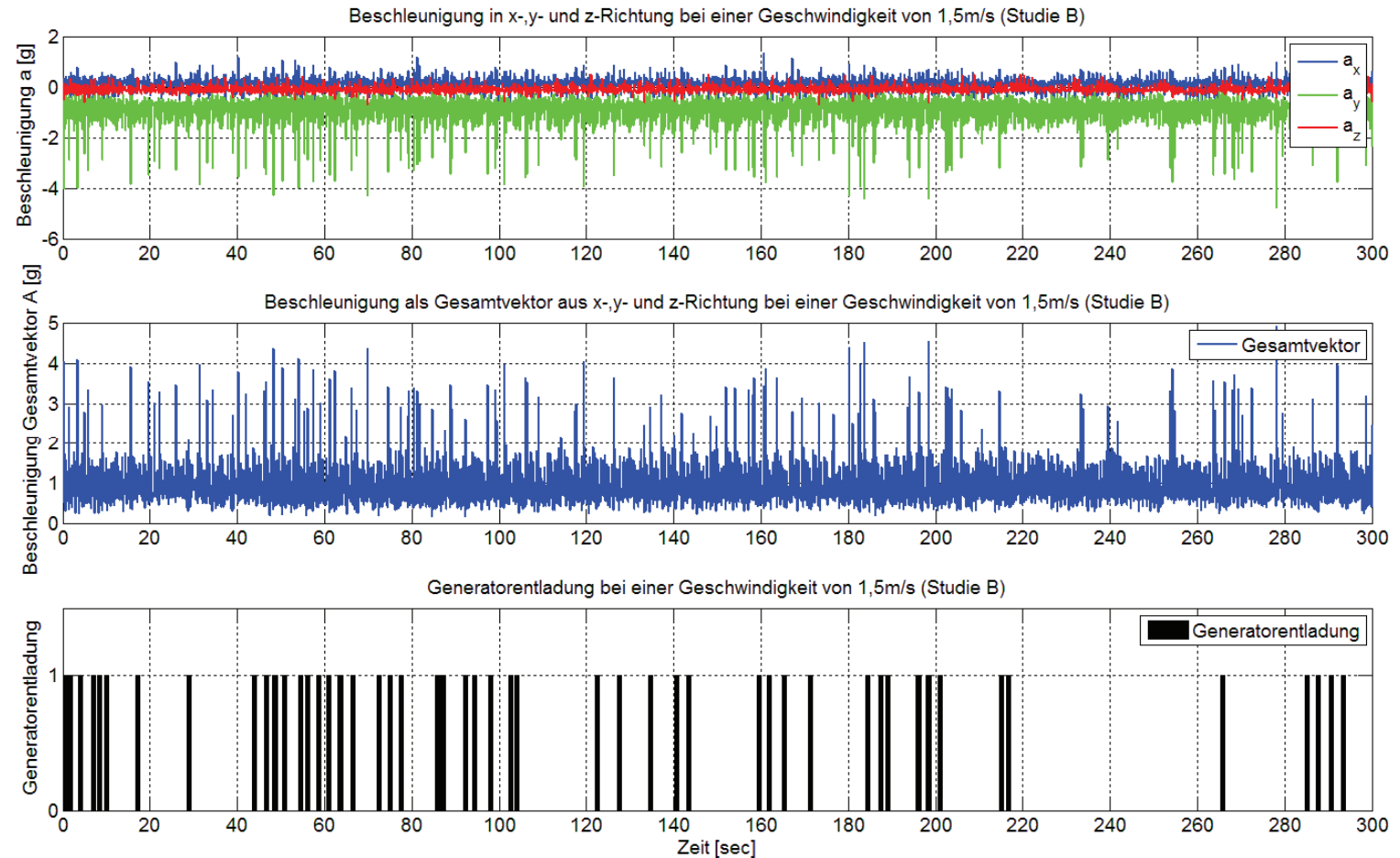

Abb. 9. Experimentelle Daten Studie B. 

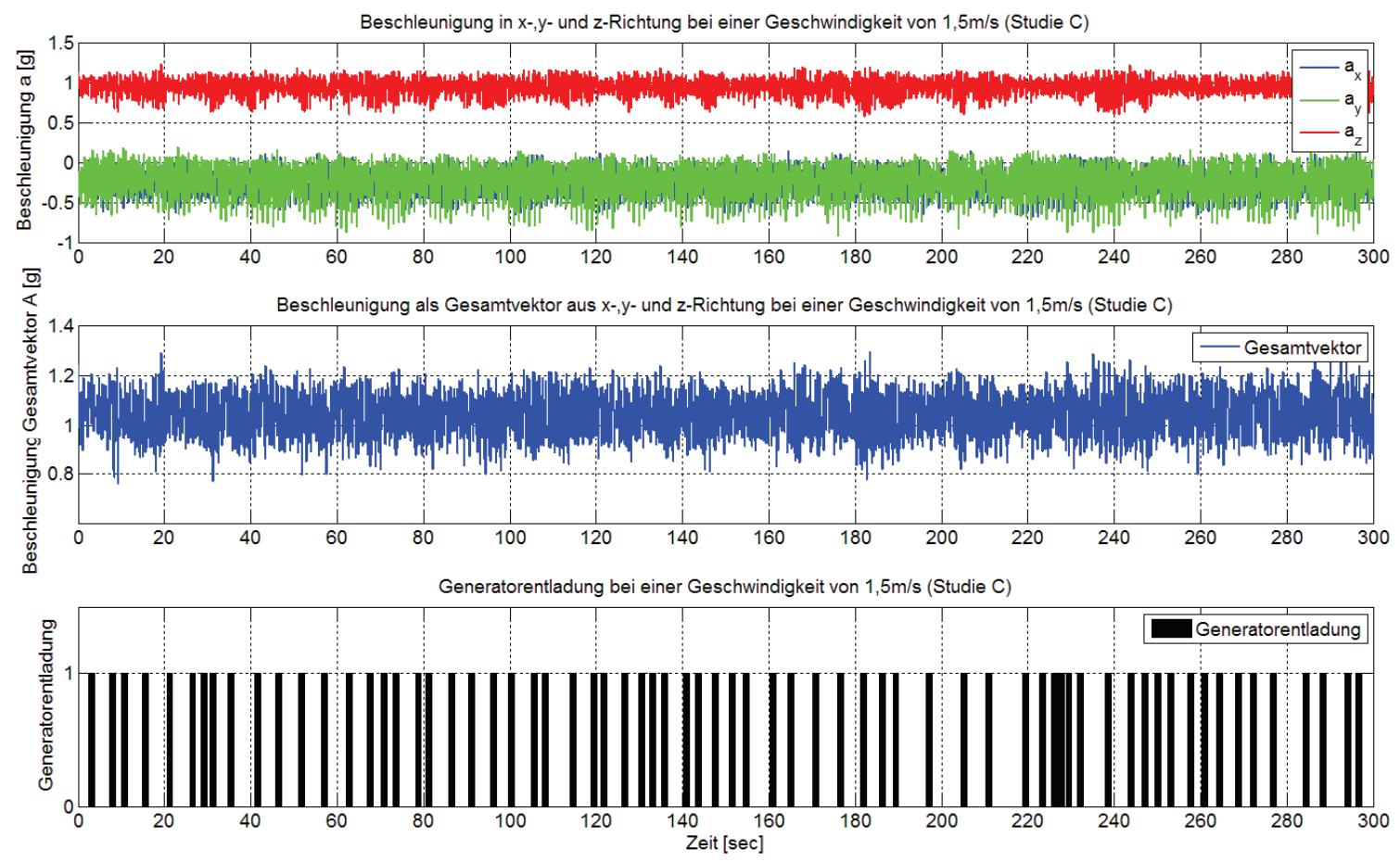

Abb. 10. Experimentelle Daten Studie C.

\section{Zusammenfassung}

Mit dem neu entwickelten Energiegenerator konnten Leistungen im $\mu \mathrm{W}$ bis $\mathrm{mW}$ - Bereich abhängig von Applikationsort und Bewegungsmuster generiert werden. Die so gewonnene Energie kann zur Unterstützung von Sensorund Telemetriesystemen, welche an Mensch und Tier Anwendung finden, beitragen. Das Ziel zukünftiger Arbeiten ist die Weiterentwicklung und Optimierung des Energiegenerators im Hinblick auf dessen Größe und Wirkungsgrad.

\section{Referenzen}

[1] T. Reuter, S. Liebold, M. Hoffmann, S. Schmidt, T. Bartsch, P. Hörschelmann, E. Weisheit, Test of a Generator for the Energy Supply of Biosensors for the Application on Human and Animal Subjects, Biomedical Engineering 56, ISSN 09394990, 2011

[2] T. Reuter, M. Beck, S. Liebold, M. Hoffmann, S. Schmidt, T. Bartsch, P. Hörschelmann, E. Weisheit, Test of a Generator in the Rumen of Cattle for Energy Harvesting to Biosensors, Biomedical Engineering 57, 572-575 (2012), ISSN (Online) 1862-278X, ISSN (Print) 0013-5585; doi: 10.1515/bmt-2012-4048

[3] O. Kanoun, J. Wallaschek, Energy Harvesting: Grundlagen und Praxis energieautraker Systeme, Haus der Technik Fachbuch Band 92, 2008, ISBN: 978-3-8169-2789-1

[4] L. Mateu, F. Moll, Review of Energy Harvesting Techniques and Applications for Microelectronics,
Proceedings of the SPIE Microtechnologies for the New Millenium, 2005

[5] W.v Engelhardt, G. Breves (Hrsg.), Physiologie der Haustiere. 15. Physiologie des Magen-DarmKanals, Enke-Verlag, Stuttgart, 2.Aufl. 2000, S. 313-422, ISBN: 3-8304-1039-5

[6] R. R. Hoffmann, B. Schnorr, Die funktionelle Morphologie des Wiederkäuermagens, Enke-Verlag, Stuttgart 1982, ISBN: 3-432-88081-2

[7] G. Dirksen, H.-D. Gründer, M. Stöber, Die klinische Untersuchung des Rindes, Enke-Verlag, Stuttgart, 4.Aufl. 2012, S. 305, ISBN: 978-3-83041211-3

[8] P. Hörschelmann, E. Weisheit, Kinetischer Wandler, DE 102011106785 A1, 2013.01.10

Danksagung: Gefördert durch das Bundesministerium für Wirtschaft und Technologie aufgrund eines Beschlusses des Deutschen Bundestages. Reg.-Nr.: KF2058703KM9

Die Autoren danken der Großtierklinik der fzmb $\mathrm{GmbH}$ für die experimentelle Unterstützung. 